\title{
Enfoques de aprendizaje en estudiantes universitarios de Buenos Aires
}

\author{
Agustín Freiberg-Hoffmann*, Mercedes Fernández-Liporace ${ }^{\star *}$, Rubén Ledesma $^{* * *}$
}

* Doctor en Psicología. Facultad de Psicología, Universidad de Buenos Aires. Becario posdoctoral, Consejo Nacional de Investigaciones Científicas y Técnicas (CONICET), Argentina. Correo electrónico: afreiberg@psi.uba.ar

** Doctora en Psicología Facultad de Psicología, Universidad de Buenos Aires. Investigadora, Consejo Nacional de Investigaciones Científicas y Técnicas (CONICET), Argentina. Correo electrónico: mliporac@psi.uba.ar

*** Doctor en Psicología. Facultad de Psicología, Universidad Nacional de Mar del Plata. Investigador, Consejo Nacional de Investigaciones Científicas y Técnicas (CONICET), Argentina. Correo electrónico: rdledesma@conicet.gov.ar

Recibido: 27 de mayo del 2016

Aprobado: 23 de noviembre del 2016

Cómo citar este artículo: Freiberg-Hoffmann,

A., Fernández-Liporace, M. y Ledesma,

R. (2017). Enfoques de aprendizaje en estudiantes universitarios de Buenos Aires. Pensando Psicología, 13(21), 17-32. doi: http://dx.doi.org/10.16925/pe.v13i21.1711

\section{Resumen}

Introducción: los enfoques de aprendizaje aluden a la predisposición para aprender en diferentes situaciones académicas. Objetivo: se analizan estos enfoques (tipos profundo y superficial) en estudiantes universitarios de Buenos Aires. Metodología: participaron 397 estudiantes de diferentes facultades (Exactas y Naturales, Psicología, Ingeniería, Derecho, Medicina) entre 18 y 36 años. Resultados: los resultados mostraron diferencias según sexo en los enfoques profundo y superficial, favorables en el primer caso a las mujeres, y en el segundo a los varones. Con respecto a la variable Facultad, se registraron varias diferencias, destacándose los estudiantes de Exactas y Naturales en el enfoque profundo, y los de Ingeniería en el superficial, en relación con el resto de las facultades. Posteriormente, se analizó la relación entre enfoques y rendimiento académico en cada facultad, verificándose asociaciones estadísticamente significativas para los grupos de Ingeniería y Medicina. Conclusiones: los resultados muestran el efecto que la variable facultad ejerce sobre los enfoques de aprendizaje, dando cuenta de la relevancia que los tipos de enseñanza adquieren en la configuración de aquellos. Las correlaciones observadas con el rendimiento académico resaltan la importancia del tipo de enfoque para ciertas carreras. Se espera que la información presentada contribuya a la planificación de actividades tendientes a mejorar el aprendizaje de contenidos por parte del estudiantado.

Palabras clave: educación superior, enfoques de aprendizaje, evaluación de estudiantes, rendimiento académico, universidades. 


\title{
Learning approaches in university students from Buenos Aires
}

\begin{abstract}
Introduction: Learning approaches allude to the predisposition to learn in different academic situations. Objective: These approaches (deep and superficial) are analyzed in university students from Buenos Aires. Methodology: 397 students between 18 and 36 years old from different schools (Exact and Natural Sciences, Psychology, Engineering, Law, and Medicine) participated. Results: The results showed differences according to sex in deep and superficial approaches, favorable to women in the first case, and to men in the second. With respect to the School variable, several differences were recorded in which Exact and Natural Sciences students stood out in the deep approach, and Engineering students in the superficial, in relation to the rest of schools. Subsequently, the relationship between approaches and academic performance in each school was analyzed, corroborating statistically significant associations for the Engineering and Medicine groups. Conclusions: Results show the effect that the School variable has on learning approaches, accounting for the relevance that teaching types have in shaping them. The observed correlations with academic performance highlight the importance of the type of approach for certain programs. It is expected that the information presented contributes to the planning of activities tending to improve students' learning of contents.
\end{abstract}

Keywords: higher education, learning approaches, student evaluation, academic performance, universities.

\section{Abordagens de aprendizagem em estudantes universitários de Buenos Aires}

\section{Resumo}

Introdução: as abordagens de aprendizagem fazem alusão à predisposição para aprender em diferentes situações acadêmicas. Objetivo: analisam-se essas abordagens (tipos profunda e superficial) em estudantes universitários de Buenos Aires. Metodologia: participaram 397 estudantes de diferentes faculdades (Exatas e Naturais, Psicologia, Engenharia, Direito, Medicina) entre 18 e 36 anos. Resultados: os resultados mostraram diferenças segundo sexo nas abordagens profunda e superficial, favoráveis no primeiro caso às mulheres e, no segundo, aos homens. A respeito da variável Faculdade, registraram-se várias diferenças, destacando-se os estudantes de Exatas e Naturais na abordagem profunda, e os de Engenharia na superficial, em relação ao restante das faculdades. Em seguida, analisou-se a relação entre abordagens e rendimento acadêmico em cada faculdade, verificando-se associações estatisticamente significativas para os grupos de Engenharia e Medicina. Conclusões: os resultados mostram o efeito que a variável faculdade exerce sobre as abordagens de aprendizagem, evidenciando a relevância que os tipos de ensino adquirem na configuração daqueles. As correlações observadas com o rendimento acadêmico ressaltam a importância do tipo de abordagem para certos cursos. Espera-se que a informação apresentada contribua para o planejamento de atividades que melhorem a aprendizagem de conteúdos por parte dos estudantes.

Palavras-chave: abordagens de aprendizagem, avaliação de estudantes, ensino superior, rendimento acadêmico, universidades. 


\section{Introducción}

Los datos actuales concernientes a la permanencia de los estudiantes en el sistema universitario resultan alarmantes. Estadísticas oficiales informan, a nivel nacional, una tasa de crecimiento anual de la población universitaria del 2,2\% entre el 2002 y el 2012 (Gobierno de Argentina-Ministerio de Educación-Secretaría de Políticas Universitarias, 2012). Paralelamente, se calcula una tasa de deserción que varía entre el 4,3\% y el 36\%, según la institución nacional que se analice (García de Fanelli, 2004; Parrino, 2008). El fracaso académico se traduce, así, en un menor número de graduados, estimado en un promedio de aproximadamente tres de cada diez alumnos ingresantes (Centro de Estudios de la Educación Argentina, 2015). El foco de la problemática se encuentra en las instancias iniciales del ciclo superior, en el cual puede apreciarse cómo, del total de ingresantes, únicamente el $20 \%$ corresponde a nuevos inscriptos, mientras que el $80 \%$ representa a alumnos que se reinscriben por diversos motivos (Gobierno de Argentina-Ministerio de Educación-Secretaría de Políticas Universitarias, 2012).

Entre los factores potencialmente intervinientes en la problemática pueden mencionarse, por un lado, los vinculados al sistema académico, así como los recursos-humanos, económicos y edilicios, entre otros, que se hallan a disposición de los estudiantes y que repercuten en sus aprendizajes (Organización de las Naciones Unidas para la Educación, la Ciencia y la Cultura, 2014). Por otra parte, se plantean las dificultades que los educandos encuentran para adecuarse a las demandas académicas de las diferentes carreras, de cara a arribar a aprendizajes eficaces y atravesar, por ende, el sistema universitario en forma exitosa (Núñez-Pérez y González-Pienda, 1993).

Teniendo estas condiciones en mente, y con la intención última de mejorarlas, investigadores locales examinan el modo en que los alumnos afrontan las diferentes situaciones de aprendizaje. Para ello, analizan distintas variables psicológicas tales como actitud, motivación, estilos de aprendizaje y aptitudes, entre otras (e. g. Corengia, Pita, Mesurado y Centeno, 2013; Montero, Pedroza, Astiz y Vilanova, 2015; Steinmann, Bosch y Aiassa, 2013; Ventura, Moscoloni y Gagliardi, 2012). En esta línea, un posible abordaje se centra en el tipo de motivación que los estudiantes manifiestan frente a las tareas académicas y las estrategias que emplean para resolverlas (Biggs, 1988). La evaluación conjunta de ambas variables se efectúa apelando al concepto enfoques de aprendizaje, el cual será abordado en la presente investigación.

\section{Enfoques de aprendizaje}

Antes de introducir el concepto de enfoques de aprendizaje, resulta útil diferenciarlo de los estilos y de las estrategias de aprendizaje con el fin de acceder a su adecuada delimitación. Los estilos describen aspectos cognitivos y de personalidad que guían las conductas que un sujeto realiza comúnmente para incorporar y articular información novedosa con la preexistente, con independencia de la situación de aprendizaje. Las estrategias, por su parte, refieren a acciones que la persona emplea con el propósito de resolver del modo más sencillo posible una tarea particular (Biggs, 1988). Estilos y estrategias corresponden a dos caras de una misma moneda, relacionándose cada estilo de aprendizaje con un conjunto de estrategias específicas (Curry, 1983). Los enfoques de aprendizaje se plantean como un constructo que analiza la flexibilidad y la capacidad que un estudiante posee para alternar el uso de diferentes estrategias en cada contexto, según cada necesidad puntual, con independencia de sus estilos de aprendizaje (Entwistle, 1988; Marton y Säljö, 1976; Schmeck, 1983). La mayor o menor versatilidad individual se asocia con el grado de comprensión que pueda alcanzar el alumno al enfrentar distintas situaciones de aprendizaje, siendo ello el objeto de interés de la presente investigación.

El análisis de los enfoques de aprendizaje permite no solo conocer el modo en que se procesa la información que debe ser aprendida (comprendiendo ello la articulación entre estilos y estrategias), sino también la intención manifiesta de encarar este proceso (Entwistle, 1991). En cuanto a esto último, el tipo de enseñanza adquiere un rol protagónico determinando el interés que los estudiantes manifestarán a la hora de enfrentarse con información novedosa (Goikoetxea-Piérola, Martínez de Lahidalga y Buján-Vidales, 2014). Así, el análisis de este constructo -a diferencia de los dos anteriores- no se centra únicamente en el educando, sino también en el tipo de enseñanza que este recibe.

A la hora de afrontar actividades de aprendizaje se pone en juego una serie de procesos metacognitivos que posibilitan la autorregulación del aprendiz. Tales procesos incluyen factores motivacionales y estratégicos que intervienen conjuntamente en la asimilación de conocimientos, guiando las acciones y el tipo de estrategias que cada alumno emplea para 
conseguir sus objetivos (Hernández-Pina, García-Sanz y Maquilón-Sánchez, 2005). El análisis de estos procesos - que integran motivos y estrategiasse efectúa a través del constructo denominado enfoques de aprendizaje (Romero Medina et al., 2013). Este concepto permite examinar la predisposición para aprender que manifiestan habitualmente los alumnos en diferentes situaciones académicas (Gargallo-López, Garfella-Esteban y Pérez-Pérez, 2006).

Comúnmente, los motivos y las estrategias se clasifican en dos categorías o tipos de enfoques denominados "superficial” y "profundo" (Marton y Säljö, 1976). El enfoque superficial es característico de aquellos estudiantes cuya motivación es estrictamente instrumental, orientada hacia la concreción de objetivos específicos - aprobar una asignatura, obtener un título, entre otros-. El principal atributo de este tipo de enfoque reside en la satisfacción de necesidades con el mínimo esfuerzo posible. La clase de estrategia relacionada con este modo de aproximarse a las situaciones de aprendizaje se basa principalmente en memorizar, retener y reproducir la información. Contrariamente, el enfoque profundo es propio de quienes se encuentran guiados por motivaciones orientadas hacia la curiosidad. Emplean estrategias tendientes a comprender la información, articulando el conocimiento nuevo con el preexistente (Biggs, 1988).

Uno de los teóricos más destacables en cuanto a sus aportes sobre este constructo es Biggs (1987), quien postula inicialmente una estructura jerárquica de los enfoques de aprendizaje. En un primer orden ubica los enfoques profundo y superficial, localizando en un segundo orden las estrategias y motivos inherentes a cada tipo de enfoque, identificados como estrategia profunda, motivo profundo, estrategia superficial y motivo superficial. Con base en esta estructura dimensional, Biggs diseña - luego de pasar por distintas versiones - el Cuestionario Revisado de Procesos de Estudio Dos Factores-Revised Two Factor Study Process Questionnaire o R-SPQ-2F (Biggs, Kember y Leung, 2001). Las evidencias de validez y confiabilidad derivadas del análisis psicométrico del instrumento han obligado al autor a postular la bifactorialidad del concepto enfoques de aprendizaje -enfoque profundo y enfoque superficial-. Esa estructura bifactorial no jerárquica resultante del estudio de Biggs et al. (2001), ha sido posteriormente puesta a prueba por distintas investigaciones que han comparado ese modelo no jerárquico versus el jerárquico. Ellas han coincidido en que el constructo se explica mejor cuando las subescalas motivación profunda y estrategia profunda, por un lado, y motivación superficial y estrategia superficial, por otro, se funden en los enfoques profundo y superficial, respectivamente. Se tiende así, actualmente - tanto por el poder explicativo, como por la mayor parsimonia verificados-, a analizar cada enfoque como una totalidad en lugar de hacerlo de manera desagregada, examinando las subescalas de motivos y estrategias (Freiberg-Hoffmann y Fernández-Liporace, 2016; Mogre y Amalba, 2014; Munshi, Al-Rukban y Al-Hoqail, 2012; Seri, Goh, Mohd y Seamah, 2010; Stes, de Maeyer y Van Petegem, 2013).

\section{Enfoques de aprendizaje en estudiantes universitarios}

El concepto enfoques de aprendizaje ha sido ampliamente investigado en estudiantes universitarios, tanto respecto de características académicas - tipo de carrera, grado de avance en la carrera, rendimiento académico-, como sociodemográficas - sexo, edad, nivel educativo de los padres- (e. g. Cetin, 2015; Cornejo y Saravia, 2014; De la Fuente, Sander y Putwain, 2013; Rajaratnam, D'cruz y Chandrasekhar, 2013; Sevsen, Senol, Mehmet y Ramazan, 2013; Mogre y Amalba, 2015; Vázquez-Martínez y Alducin-Ochoa, 2014; Wong, Kwong y Thadani, 2014; Zhang, 2000).

El éxito académico suele asociarse con el enfoque profundo, representado por la comprensión del contenido que se desea aprender en situaciones específicas (Bernardo, 2003; Biggs y Tang, 2011; Entwistle y Ramsden, 1983). No obstante, se destaca la importancia del enfoque superficial frente a determinadas tareas académicas. Por caso, se ha reportado cómo la memorización y reproducción de conceptos pueden, en ocasiones, beneficiar el desempeño estudiantil (Kember, Leung y McNaught, 2008; Smith y Miller, 2005).

Según se afirma, la eficacia del tipo de enfoque depende en buena medida de las características vinculadas al contexto y a la modalidad de instrucción. Así, se destaca que un estudiante es más proclive a adoptar un enfoque profundo cuando se enfrenta a situaciones de aprendizaje que requieren para su solución de análisis crítico y comprensión. En cambio, se elige el superficial cuando se participa de contextos tradicionales de aprendizaje, en los que se proponen actividades académicas que deben ser abordadas por los educandos 
mediante acciones estandarizadas y memorísticas (Sadlo y Richardson, 2003; Wang, Su, Cheung, Wong y Kwong, 2013). Esto se condice con el papel que el individuo asume en cada situación. En la primera modalidad de aprendizaje se ubica el acento en la resolución activa de problemas. En la segunda, en cambio, el estudiante se convierte en partícipe pasivo, interesándose únicamente en cumplir con el plan preestablecido antes que en comprender los conocimientos que debe incorporar (Marton, 1976). En este punto, resulta interesante destacar que ambos tipos de enfoques representan dos modos diametralmente opuestos de aprender que conviven en cada estudiante, ya que no todas las tareas académicas requieren, para ser abordadas eficazmente, del empleo de un mismo tipo de enfoque. Esto lleva a los educandos a la necesidad de fluctuar, según el tipo de actividad, desde un enfoque hacia el otro de modo permanente. Así, la frecuencia de uso de cada enfoque dependerá de la clase de tareas que requiera cada disciplina académica en particular (Argos, Ezquerra, Osoro, Salvador y Castro, 2013).

Si bien localmente es la modalidad instruccional tradicional la que prima, también es cierto que los docentes intentan paulatinamente incorporar actividades que estimulen la flexibilidad a la hora de resolver situaciones problemáticas. Tal inclusión es variable según la disciplina, adoptando los alumnos el enfoque que mejor se ajuste a cada caso. En este sentido, la motivación y las estrategias que aquellos manifiestan en cada carrera se encuentran relacionadas con la modalidad de evaluación empleada en la mayoría de las asignaturas que las integran. Consecuentemente, las disciplinas que evalúan el rendimiento de los alumnos a través de un método vinculado con la memorización de conceptos, datos, o bien con la resolución algorítmica de problemas, premian con buenas calificaciones a quienes emplean un enfoque superficial. Al contrario, las especialidades que valoran los conocimientos adquiridos mediante procedimientos que admiten cierto grado de flexibilidad en las respuestas suelen trabajar con el tipo profundo (González, 1997; Novak, 1977).

Esta relación dialéctica entre el tipo de enfoque a adoptar y la modalidad de instrucción, suele ser un obstáculo que deben resolver los estudiantes que inician sus trayectorias universitarias. Para ello, necesitan destinar una parte considerable de su tiempo a adaptar sus habilidades a fin de aprender al modo requerido por la carrera elegida. Esto se traduce, en ciertos casos, en inconvenientes para asimilar los conocimientos en esta instancia inicial, llevando a extender los tiempos establecidos hasta la graduación (Zain, Malan, Noordin y Abdullah, 2013). En ocasiones, la adaptación se realiza en tiempo y forma según el plan de estudios, en tanto que en otras la dificultad para adecuarse a las nuevas modalidades de aprendizaje se traduce en situaciones de fracaso académico, produciéndose reinscripciones o abandonos. El tipo de procesamiento metacognitivo que demandan las distintas actividades de aprendizaje de cada especialidad académica se erige, así, en un indicador clave que permite identificar las habilidades que facilitarían la asimilación de conocimiento en cada una de aquellas.

El presente trabajo analiza los enfoques de aprendizaje en estudiantes de distintas facultades. Desde el punto de vista aplicado, conocer este atributo en profundidad al interior de cada unidad académica y/o de cada grupo áulico puede resultar de suma utilidad para planificar actividades docentes, tendientes a facilitar la asimilación de conocimientos por parte del estudiantado. De esta manera, los docentes pueden adecuar sus prácticas según el tipo de enfoque predominante en los alumnos, articulando tareas basadas en la resolución de problemas con otras más tradicionales. Paralelamente, las instituciones pueden reestructurar sus planes de estudio - orden de las asignaturas, cargas horarias y prácticas profesionales, entre otros- adecuándolos a las características de los educandos. Finalmente, esta información interesa a los propios estudiantes, quienes, a partir de intervenciones llevadas a cabo por los profesionales de la educación, pueden lograr autorregular sus aprendizajes seleccionando las habilidades y estrategias más apropiadas y así conseguir el logro de sus objetivos.

Resumiendo, se espera así que esta información contribuya fundamentalmente a la detección precoz de casos en potencial riesgo académico, para luego arbitrar los medios necesarios (institucionales y pedagógicos, entre otros), tendientes a prevenir el fracaso estudiantil que actualmente preocupa a autoridades universitarias.

Se plantea abordar los siguientes objetivos: a) analizar posibles diferencias estadísticamente significativas en los enfoques de aprendizaje (superficial y profundo), según variables sociodemográficas (sexo, edad y facultad); b) analizar la relación entre los enfoques de aprendizaje (superficial y profundo), y el rendimiento académico en los estudiantes de distintas facultades. 


\section{Método}

\section{Diseño}

Se empleó un diseño transversal, correlacional, de diferencias entre grupos y explicativo. Los datos fueron recogidos mediante un muestreo por conveniencia (Miles y Banyard, 2007; Scheaffer, Mendenhall y Ott, 2007).

\section{Instrumentos}

\section{Encuesta sociodemográfica y académica}

Variables sociodemográficas relevadas: sexo, edad.

Variables académicas: facultad, número total de asignaturas aprobadas, año de inicio de estudios en la facultad. Las dos últimas fueron incluidas a fin de estimar una medida del rendimiento académico (en el apartado de procedimientos se detalla la construcción del índice de rendimiento académico mediante el empleo de ambos indicadores). Debe mencionarse que este modo de medir el desempeño estudiantil es restringido y parcial, y representa solo una forma posible, muy sencilla y lineal, de aproximarse a la evaluación del fenómeno. De ningún modo tal estimación constituye una representación fiel del rendimiento académico abarcado en su totalidad, dada la complejidad que el concepto entraña. Tal concepto, por ejemplo, incluye - en un sentido amplio- aspectos tales como satisfacción respecto de la carrera, tasa de deserción, duración media de los estudios, tasa de eficiencia y tasa de éxito, entre otros (Martín, García, Torbay y Rodríguez, 2008). Por esta razón, la operacionalización elegida para este estudio representa un recorte muy particular de aquel, y debe ser interpretado con las reservas del caso. El criterio utilizado para su elección se corresponde, sencillamente, con la medida que las instituciones universitarias locales adoptan para establecer la condición de regularidad de los estudiantes exigiendo, como mínimo, la aprobación de dos asignaturas por año (Gobierno de Argentina-Ministerio de Educación, 1995).

Cuestionario Revisado de Procesos de Estudio, Dos Factores (R-SPQ-2F según Biggs et al. (2001)

Se aplicó la versión adaptada del instrumento para estudiantes universitarios de Buenos Aires (Freiberg-Hoffmann y Fernández-Liporace, 2016). Evalúa los enfoques de aprendizaje profundo y superficial a través de veinte ítems (diez por cada dimensión), con escala de respuesta Likert de cinco opciones. El instrumento cuenta con evidencias de validez de contenido, aparente y de constructo (estudios factoriales exploratorio y confirmatorio). Las dimensiones derivadas del proceso de adaptación presentan similar relevancia, explicando el enfoque superficial un $27,9 \%$, y el profundo un $20,9 \%$, de la varianza común, totalizando un $48,8 \%$. Por otra parte, esta versión da cuenta de la ortogonalidad de los factores extraídos, indicando ello la independencia de los enfoques. El cuestionario presenta, además, adecuados índices de confiabilidad test-retest $(r>0,70)$, y de consistencia interna $\left(\alpha_{\text {ordinal }} \geq 0,70\right)$ para cada una de sus dimensiones. La consistencia interna fue verificada también en la presente muestra, replicando los valores obtenidos en el estudio local previo; precisamente, para el enfoque superficial se alcanzó un alfa ordinal de 0,85 , mientras que para el profundo, 0,77 .

\section{Participantes}

La muestra se conformó por 397 estudiantes universitarios del área metropolitana de Buenos Aires (56,7\% varones; 43,3\% mujeres), provenientes de distintas facultades (24,7\% Exactas y Naturales; $23,9 \%$ Psicología; 22,4\% Ingeniería; 17,6\% Derecho; y 11,3\% Medicina). A fin de asegurar la representatividad de los datos, la recolección de los mismos se realizó en las tres universidades que registran la mayor matriculación de estudiantes del área metropolitana según estadísticas oficiales, siendo esta matriculación masiva y significativamente mayor respecto del resto de las universidades de la zona (Gobierno de Argentina-Ministerio de Educación-Secretaría de Políticas Universitarias, 2012). Las edades variaban entre 18 y 36 años $\left(_{\text {Edad }}=\right.$ $23,81 ; \mathrm{DE}=3,24)$. Se excluyeron, por otro lado, los estudiantes que se encontraban cursando el primer año del ciclo universitario. Este criterio responde a la necesidad de trabajar con muestras de educandos con, al menos, un año de permanencia en la orientación por ellos elegida inicialmente, ya que según se informa, el $58 \%$ del total de estudiantes que cursan el primer año abandona los estudios o cambia de carrera (Pintos, 2012).

\section{Análisis de datos}

Los datos fueron analizados a través del programa SPSS 21 (Iвм Corporation, 2012). Se empleó, a fin de 
responder a los distintos objetivos, el análisis multivariado de la covarianza (MANCOVA), y el análisis correlacional $r$ de Pearson. Adicionalmente, se realizaron estimaciones del tamaño del efecto (TE) siguiendo los criterios de interpretación propuestos por Cohen (1977).

\section{Procedimientos}

El estudio contó con los avales institucionales correspondientes. Los datos fueron recogidos por un psicólogo matriculado durante el horario habitual de clases. La participación de los estudiantes fue voluntaria, con la posibilidad explícita de cesar de responder en cualquier instancia de la administración. No se ofreció retribución económica ni académica, y se firmó un consentimiento informado, garantizando la confidencialidad de los resultados y el anonimato de los datos.

Previo al cálculo del MANCOVA se procedió a analizar el cumplimiento de algunos supuestos que habilitan su ejecución. Se revisó, primero, la homogeneidad de covarianzas, que resultó verificada ( $\mathrm{M}$ de Box $=49,396 ; p=0,008)$, de acuerdo con Huberty y Petroskey (2000). Luego se examinó la homogeneidad de varianzas, la cual se verificó solo para una de ambas variables dependientes $\left(p_{\text {profundo }}=0,153 ; p_{\text {superficial }}<\right.$ $0,001)$, llevando ello a utilizar para el análisis el estadístico Traza de Hotelling, considerado robusto en casos de heterocedasticidad (Finch y French, 2013). Posteriormente, se examinó el cumplimiento del supuesto de multicolinealidad entre variables dependientes $(r=-0,381 ; \mathrm{p}<0,01)$, el cual quedó verificado (Tabachnick y Fidell, 2013).

Por otra parte, en el análisis correlacional entre los enfoques y el rendimiento académico se estimó el desempeño estudiantil (variable cuantitativa), calculando el cociente entre el número de asignaturas aprobadas y la antigüedad en años que lleva cada estudiante en el sistema universitario (De Miguel y Arias, 1999).

\section{Resultados}

El análisis MANCova tomó los enfoques de aprendizaje profundo y superficial como variables dependientes, por un lado, y sexo y facultad como factores, por el otro. La edad se tomó como covariable. Los resultados mostraron efectos multivariados significativos para las variables sexo $\left(\mathrm{F}_{(2,385)}=11,029 ; p=0,000\right.$; $\left.\eta^{2}=0,054\right)$ y Facultad $\left(\mathrm{F}_{(8,768)}=2,772 ; p=0,005 ; \eta^{2}=\right.$ $0,028)$ sobre los enfoques de aprendizaje. Los valores similares de Traza de Hotelling (0,057 para sexo y 0,058 para Facultad), dan cuenta del efecto parejo que ambas variables tienen sobre los enfoques. Al examinar las medias se observó que las diferencias para el enfoque profundo fueron favorables a las mujeres varones $_{(n=225)}=32 ; \mathrm{DE}=5,90 ;$ mujeres $_{(n=172)}=$ $33,25$; $\mathrm{DE}=4,53)$, mientras que los varones prevalecieron en el superficial (varones $_{(n=225)}=23,25 ; \mathrm{DE}=$ 6,74 ; $\left._{\text {mujeres }}^{(n=172)}=18,91 ; \mathrm{DE}=5,29\right)$.

Con respecto a la variable Facultad, se registró una diferencia favorable a los estudiantes de Exactas y Naturales en comparación con los de Derecho e Ingeniería en el enfoque profundo. En cambio, para el superficial se verificó, por un lado, una diferencia favorable a los estudiantes de Ingeniería en comparación con los del resto de las Facultades (Psicología, Medicina, Exactas y Naturales, Derecho), y por otro, otra diferencia entre los estudiantes de Psicología, Derecho y Exactas y Naturales, a favor de los dos últimos. Las diferencias registradas presentaron un tamaño del efecto bajo, tanto para el enfoque profundo, como para el superficial (véase la tabla 1).

Tabla 1

Enfoques de aprendizaje. Análisis de diferencias entre facultades

\begin{tabular}{lccc}
\hline \multicolumn{1}{c}{ Facultad } & & \multicolumn{2}{c}{ Enfoques de aprendizaje } \\
& & Profundo & Superficial \\
\hline $\begin{array}{l}\text { Psicología } \\
(n=95)\end{array}$ & $\bar{x}$ & $33,23^{\mathrm{AB}}$ & $18,47^{\mathrm{A}}$ \\
& $\mathrm{DE}$ & 4,94 & 5,72 \\
\hline $\begin{array}{l}\text { Medicina } \\
(n=45)\end{array}$ & $\bar{x}$ & $32,24^{\mathrm{AB}}$ & $20,04^{\mathrm{AB}}$ \\
\hline $\begin{array}{l}\text { Exactas y Naturales } \\
(n=98)\end{array}$ & $\bar{x}$ & $34,19^{\mathrm{B}}$ & 5,32 \\
\hline $\begin{array}{l}\text { Derecho } \\
(n=70)\end{array}$ & $\mathrm{DE}$ & 5,29 & $21,11^{\mathrm{B}}$ \\
& $\bar{x}$ & $31,51^{\mathrm{A}}$ & 5,55 \\
\hline $\begin{array}{l}\text { Ingeniería } \\
(n=89)\end{array}$ & $\mathrm{DE}$ & 5,23 & $21,77^{\mathrm{B}}$ \\
& $\bar{x}$ & $30,95^{\mathrm{A}}$ & 6,41 \\
\hline F & $\mathrm{DE}$ & 5,92 & $25,13^{\mathrm{C}}$ \\
$p$ valor & & 2,573 & 7,16 \\
\hline$\eta^{2}$ & & 0,037 & 3,347 \\
\hline
\end{tabular}

Nota. Letras diferentes indican diferencias estadísticamente significativas entre los grupos. ANOva con contraste post-hoc Tukey. Elaboración propia. 
Por otra parte, no se registró un efecto estadísticamente significativo, tanto de la covariable edad, como de la interacción entre los factores sexo y facultad, sobre los enfoques de aprendizaje.

Luego del análisis de diferencias se procedió a estudiar la relación entre cada enfoque de aprendizaje y el rendimiento académico al interior de cada facultad. La tabla 2 resume las asociaciones para todas las facultades, de manera que se puede apreciar que solo las de Ingeniería y Medicina registraron relaciones estadísticamente significativas.

Tabla 2

Correlaciones entre enfoques de aprendizaje y rendimiento académico por facultad

\begin{tabular}{lcc}
\hline Rendimiento Académico / Facultad & \multicolumn{2}{c}{ Enfoques de aprendizaje } \\
\cline { 2 - 3 } & Profundo & Superficial \\
\hline $\begin{array}{c}\text { Ingeniería } \\
(n=89)\end{array}$ & $-0,296^{* *}$ & $0,308^{* *}$ \\
$\begin{array}{c}\text { Medicina } \\
(n=45)\end{array}$ & & $-0,296^{*}$ \\
\hline
\end{tabular}

Nota. ${ }^{*} \mathrm{p}<0,01 ;{ }^{* *} \mathrm{p}<0,05$. Elaboración propia.

\section{Discusión}

El presente estudio analiza los enfoques de aprendizaje en estudiantes universitarios, centrando el interés en conocer la predisposición para aprender que poseen los educandos de diferentes facultades. Primero, se analizaron los enfoques según variables sociodemográficas, observándose diferencias según sexo, tanto en el tipo superficial, como en el profundo, favorable el primero a los varones, y el segundo a las mujeres. Estos resultados van en el mismo sentido que los reportados por investigaciones previas (e. g. García-Berbén, 2005; Marrs y Sigler, 2012). De acuerdo con esto, es posible inferir que los varones tienen una mayor inclinación a explorar los textos de manera superficial, sin intentar articulaciones conceptuales. Para ello emplearían estrategias orientadas a la selección de la información relevante, su memorización y posterior reproducción en la instancia de evaluación. Estos sujetos presentan una motivación instrumental que los lleva a considerar el estudio como un medio para acceder a un fin (la graduación, inserción en el medio laboral). Contrariamente, las mujeres se encontrarían más motivadas por la curiosidad, mostrando especial interés por la aprehensión de los contenidos previstos en las asignaturas.
Estudiarían, por ende, con el propósito de satisfacer su deseo de conocimiento, interesadas por entender significados conceptuales y su relación con otras nociones teóricas. Sus estrategias, así, se infieren basadas en la lectura y discusión de los distintos temas de estudio, arribando a la formulación de hipótesis acerca del modo en que las ideas pueden articularse (Biggs, 1991).

En lo que respecta a las variables académicas, el análisis de diferencias reveló que los estudiantes que más frecuentemente emplean el tipo de enfoque profundo provienen de la Facultad de Ciencias Exactas y Naturales, mientras que los de Ingeniería se destacan por encima del resto en el uso del enfoque superficial. Estos resultados se mueven en la misma dirección que los informados por otros autores (e. g. Byrne, Finlayson, Flood, Lyons y Willis, 2010; Ng y Ng, 1997; Tarabashkina y Lietz, 2011). De acuerdo con esto, quienes cursan carreras exactas y naturales prefieren asimilar la información realizando un arduo trabajo de lectura, interpretación y comprensión de los contenidos de cada asignatura. Estos estudiantes resolverían los diferentes problemas (evaluación u ejercicios prácticos) razonando a partir de distintos principios para llegar, por alguno de los caminos posibles, al resultado esperado. Contrariamente, los estudiantes de Ingeniería parecen elegir aprender mediante la detección de los contenidos más importantes y su memorización o automatización, para luego reproducirlos en la instancia de evaluación. Los alumnos de esta facultad abordarían las tareas mediante la aplicación de fórmulas y algoritmos, centrando la prioridad en la selección del procedimiento correcto para la solución de un problema específico.

Pasando ahora al estudio correlacional entre rendimiento académico y enfoques de aprendizaje, se observa en estudiantes de Ingeniería que, a medida que aumenta el rendimiento, decrece el enfoque profundo y se incrementa el superficial. Este resultado se vuelve lógico al considerar que el análisis de diferencias da cuenta de un mayor uso del enfoque superficial en los educandos de este grupo, como cualidad distintiva del resto de los estudiantes de las otras facultades analizadas. Si bien se considera que el aprendizaje eficaz se asocia con el enfoque profundo, es necesario recordar la influencia que el contexto tiene sobre los enfoques (Sadlo y Richardson, 2003; Wang et al., 2013). Así, los estudiantes tienden a adecuarse al tipo de enseñanza que reciben. Esto permite hipotetizar, según Biggs y Tang (2011), que el grupo de Ingeniería se hallaría frente a una modalidad instruccional caracterizada 
por tiempos más limitados para la resolución de tareas en clase, una oferta de material de estudio basada en fuentes secundarias de información (resúmenes, diapositivas, entre otros) con antelación a la consulta de fuentes primarias, y una metodología de evaluación centrada en la reproducción de información y procedimientos, en la cual la articulación conceptual no es la tendencia predominante. Este supuesto permitiría explicar cómo, además de erigirse el enfoque superficial en una característica destacable, en ellos se asocia también con mejores rendimientos en su caso, llevándolos a una mayor eficacia académica, siendo el enfoque profundo menos útil en este sentido. Como antes se ha destacado, el costo que esta modalidad de enseñanza puede pagar es el de generar en algunos alumnos la necesidad de recompensas extrínsecas, lo cual los lleva a desear aprobar las asignaturas simplemente con el afán de graduarse, estudiar de memoria para conseguir tal fin, y priorizar actividades extraacadémicas por sobre las académicas, entre otras características destacables.

Contrariamente, los estudiantes de Medicina observan una relación estadísticamente significativa entre el rendimiento académico y el enfoque superficial, aunque negativa. Ello quiere decir que, a medida que el enfoque superficial decrece, el desempeño mejora. Sin embargo, al no mostrar este grupo diferencia con respecto del resto de las facultades en el empleo de un enfoque $u$ otro, se torna imposible distinguir el perfil predominante, llevando a pensar que, tal vez, el uso combinado de ambos enfoques sea más útil para ellos. No obstante, debido a que el rendimiento ha sido estimado en función del número de asignaturas aprobadas, se vuelve factible suponer que la modalidad de evaluación mayormente utilizada en ellas no presentaría un formato beneficioso para quienes usen el enfoque superficial.

En cuanto al resto de las facultades analizadas (Psicología, Exactas y Naturales, Derecho), no se han logrado verificar asociaciones estadísticamente significativas entre rendimiento académico y enfoques de aprendizaje. Este resultado se orienta en la misma dirección que otras investigaciones, la cuales concluyen que, o bien el modo en que los estudiantes se aproximan a las situaciones de aprendizaje no constituye un factor determinante del desempeño académico, o bien el tipo de acercamiento no tiene un efecto directo sobre el rendimiento, sino más bien indirecto (Al-Qahtani, 2015; Karagiannopoulou y Milienos, 2015). No obstante, dada la evidencia obtenida en este trabajo en las facultades de Ingeniería y Medicina, así como también la derivada de otros estudios que sí han hallado tal relación, resulta conveniente continuar indagando este punto en futuros desarrollos (Hasnor, Ahmad y Nordin, 2013; Salamonson et al., 2013; Svedin, Balter, Scheja y Pettersson, 2013). Podría entonces analizarse tal asociación en otras unidades académicas no contempladas en este estudio, o bien segmentando por carreras, o tal vez reagrupando las facultades en categorías mayores, como, por ejemplo, las ramas de estudio (ciencias aplicadas, básicas, de la salud, humanas, sociales), propuestas por el Gobierno de Argentina, Ministerio de Educación-Secretaría de Políticas Universitarias (2012).

Queda, por otro lado, pendiente un análisis de la relación entre enfoques de aprendizaje y rendimiento académico, el cual contemple la evaluación de efectos conjuntos de ambos enfoques. Esto no ha podido analizarse en este trabajo por no disponerse de un tamaño muestral apropiado. Asimismo, sería de interés complementar esta información con datos vinculados a los tipos de enseñanza que los estudiantes reciben.

El principal aporte de esta investigación se centra en la caracterización de los estudiantes de distintas facultades en cuanto a los enfoques de aprendizaje. Además, se informa sobre la relación que tales enfoques mantienen con el rendimiento académico, sin implicar ello necesariamente que los enfoques influyan positiva o negativamente sobre el desempeño estudiantil. Por el contrario, investigaciones recientes sostienen que los educandos deben desarrollar ambos tipos de enfoques, así como también la capacidad de decidir correctamente el empleo de uno u otro en relación al tipo de actividad que deben afrontar (Argos et al., 2013; Goikoetxea-Piérola et al., 2014). El uso incorrecto de un enfoque en la resolución de una tarea específica probablemente se asocie con un resultado negativo. No obstante, ello no quiere decir que el tipo de enfoque sea prejudicial en sí mismo, sino que ha sido seleccionado equivocadamente para resolver una actividad dada. La situación se agrava aún más cuando el estudiante intenta abordar mediante un solo tipo de enfoque todas las instancias académicas de la carrera que cursa, sobre todo cuando dicha disciplina plantea situaciones que requieren soluciones dependientes del tipo de enfoque opuesto. Por esta razón, las propuestas actuales abogan por fomentar el desarrollo de ambos enfoques de aprendizaje en los estudiantes y, complementariamente, por entrenar la capacidad individual que les permita discernir el momento apropiado para emplearlos. 
Con relación a las limitaciones de esta investigación, debe mencionarse que no se han incluido en la muestra aquellos alumnos que se encontraban cursando el primer año del ciclo universitario, inclusión que hubiera permitido establecer comparaciones entre estudiantes ingresantes y avanzados de las facultades analizadas. En este sentido, estudios que sí han realizado tal comparación verificaron que, en instancias iniciales, los alumnos tienden a manifestar un enfoque predominantemente superficial, mientras que los avanzados muestran preponderancia del tipo profundo (e. g. Hasnor et al., 2013; Salim y Lotti de Santos, 2011). Dada la importancia de esta clase de examen, este se abordará en futuros trabajos, con especial interés en cuanto al diseño o reforma de programas de estudio.

Por otra parte, en cuanto a las asociaciones derivadas del análisis correlacional, debe destacarse que - si bien son bajas - pueden ser admitidas, ya que los valores habituales dentro del campo de investigación en psicología rondan coeficientes de 0,30 (Hemphi1l, 2003). Estos guarismos, no obstante, podrían incrementarse si la muestra se segmentara por carrera, ya que la mayor especificidad podría aumentar estas magnitudes. En la presente investigación se ha decidido agrupar a los estudiantes por facultad, ya que la división por carrera habría llevado a la formación de una mayor cantidad de grupos con tamaños muy heterogéneos, dificultando los análisis. En este sentido, no puede soslayarse la cuestión concerniente al tamaño muestral que afecta el $n$ de los grupos segmentados según facultad. Si bien se han adoptado los recaudos metodológicos que aseguran la robustez de los resultados, es probable que los niveles de significación mejoren al incrementar los $n$ grupales que representan a cada una de las unidades académicas incluidas. Futuros estudios se encargarán de mejorar esta limitación mediante la recolección de datos, correspondientes a mayor cantidad de alumnos de las facultades analizadas en este trabajo.

Recapitulando, el tipo de enfoque que adoptan los estudiantes se encuentra estrictamente relacionado con el contexto educativo del que participan. Así, los educandos con un enfoque profundo responden a un contexto orientado hacia la resolución activa de problemas, mientras que los superficiales prefieren contextos de tipo tradicional, basados en el cumplimiento de planes de estudio y en la aprobación de asignaturas en tiempo y forma (Sadlo y Richardson, 2003; Wang et al., 2013). En este punto es vital destacar que ambos enfoques son igualmente necesarios, ya que su importancia radica en el tipo de contenido a ser aprendido. En términos generales, quienes deben asimilar información que requiera ser memorizada antes que comprendida - adquirir un mayor vocabulario, aprender el papel de una obra de teatro, o aprender una fórmula-, emplearán predominantemente un tipo de enfoque superficial. En cambio, quienes deban otorgar sentidos y comprender la información a asimilar - aprender desarrollos matemáticos, teoremas, principios, una teoría, o hechos históricos-, observarán un uso acentuado del enfoque profundo (Biggs y Tang, 2011). De todos modos, aquellos que deban memorizar parte de una obra a fin de interpretarla actoralmente, la interpretarán cabalmente solo si logran la integración de ambos enfoques: el superficial, para memorizar letras, actitudes, tiempos y desplazamientos; y el profundo, con el propósito de comprender la esencia del personaje en relación con la trama y el resto de los personajes, llegando así a una producción final de calidad. Ejemplos como este pueden pensarse al infinito para todas las carreras y situaciones de aprendizaje, de recuperación y puesta en juego de los conocimientos, así como para el futuro desempeño profesional.

Volviendo a los resultados en sí, parecería que, las mujeres por un lado, y los alumnos de la Facultad de Ciencias Exactas y Naturales, por otro, exhiben un acercamiento mayormente profundo hacia el conocimiento, implicando ello la preferencia por situaciones educativas basadas en la resolución activa de problemas. Existen ciertas prácticas pedagógicas tendientes a fomentar este enfoque en aquellos estudiantes que lo presentan en baja medida. Entre las más importantes se puede mencionar el planteo de actividades lúdicas o motivadoras que generen en los educandos el interés por aprender, lo cual los llevé a comprometerse de un modo diferente con las tareas a afrontar. En esta misma dirección debe estimularse el trabajo colaborativo y el diálogo entre pares mediante diferentes actividades, puesto que ello puede mejorar sustancialmente la comprensión de temas diversos (Biggs y Tang, 2011).

Por otro lado, estudiantes varones o que cursan en la Facultad de Ingeniería, manifiestan un enfoque predominantemente superficial, el cual se condice con una mayor comodidad frente a contextos educativos tradicionales. Pueden instrumentarse, también para esta clase de enfoque, ciertas prácticas educativas orientadas a su estimulación. Se destaca así la propuesta de una modalidad de trabajo de tipo individual, con clases expositivas en las que el docente presenta 
diferentes conceptos y sus relaciones, eligiendo una menor profundización en aras de brindar una mayor amplitud de información. Otra práctica muy común es la de proporcionar a los estudiantes una amplia cantidad de fuentes de información secundaria - fichas de estudio elaboradas por el docente, presentaciones audiovisuales, apuntes tomados en clase, entre otras-, que sirvan como material de estudio, con antelación a la consulta de textos originales (Biggs y Tang, 2011).

De todos modos, la importancia de tender a la integración de ambos enfoques no debe jamás soslayarse. Las instituciones y los docentes debieran instrumentar programas y estrategias educativas claros y definidos, dirigidos a la construcción de la capacidad individual que permita integrar - o bien virarsegún el caso y la necesidad, desde un enfoque hacia el otro, de acuerdo con las problemáticas y contenidos presentados, y de cara a la transferencia de tal versatilidad en un momento posterior a situaciones laborales. Sin embargo, para ello antes deben las instituciones y los propios docentes tomar conciencia de estas necesidades, flexibilizando sus propios estándares y prácticas instituidas.

Se destaca la contribución de este trabajo en la intención de aportar información de utilidad para la psicología educacional y el ámbito educativo aplicado, facilitando la detección temprana de los enfoques de aprendizaje en el alumnado universitario. A partir de ello, los profesionales psicólogos de la educación estarían en condiciones de diagramar intervenciones destinadas a estimular aquellos aspectos motivacionales y estratégicos que cada estudiante presentare en baja medida, beneficiando - eventualmente- los procesos de aprendizaje según el caso. Por otro lado, dicha estimulación debería complementarse con una correcta capacitación de los estudiantes en la identificación de las distintas actividades académicas y la adecuada adopción del tipo de enfoque que facilite su abordaje, ya que no todas las tareas son susceptibles de ser resueltas del mismo modo. En esta misma dirección debe destacarse también el rol de los docentes, quienes podrían proponer nuevas modalidades de evaluación que contemplen de parte del alumno la utilización de ambos tipos de enfoques. Así, el educando deberá primeramente identificar la naturaleza de la tarea a resolver, y elegir, en consecuencia, el enfoque más pertinente. Por otra parte, a nivel institucional las autoridades universitarias pueden tomar decisiones en torno a la modificación de los planes de estudio, a fin de integrar asignaturas cuyas propuestas académicas dependan de enfoques de aprendizaje diferentes, evitando que el excesivo empleo de un enfoque en una instancia académica perjudique el desarrollo de otro.
Esta tarea mayor debe ser encarada mediante equipos interdisciplinarios integrados por pedagogos, especialistas en didáctica especial, psicólogos, psicopedagogos, profesores de cada área y carrera y especialistas en gestión de la educación superior.

Nuevas investigaciones profundizarán estos análisis al interior de cada unidad académica y en diferentes momentos del ciclo, de modo tal que sea posible conocer con mayor precisión la evolución de los enfoques durante los trayectos universitarios, su relación con el rendimiento estudiantil y con el aprendizaje eficaz.

\section{Referencias}

Al-Qahtani, M. (2015). Association between approaches to study, the learning environment, and academic achievement. Journal of Taibah University Medical Sciences, 10(1), 56-65. doi: 10.1016/j.jtumed.2015.01.014

Argos, J., Ezquerra, P., Osoro, J., Salvador, L. y Castro, A. (2013). La evaluación de los aprendizajes de los estudiantes en el marco del Espacio Europeo de Educación Superior (EEEs): sus prácticas, preferencias y evolución. European Journal of Investigation in Health, 3(3), 181-194. doi: 10.1989/ejihpe.v3i3.41

Bernardo, A. (2003). Approaches to learning and academic achievementofFilipinostudents.JournalofGeneticPsychology, 164(1), 101-114. doi: 10.1080/00221320309597506

Biggs, J. (1987). Student approaches to learning and studying. Melbourne: Australian Council for Educational Research.

Biggs, J. (1988). Assessing study approaches to learning. Australian Psychologist, 23, 197-206. doi: 10.1080/ 00050068808255604

Biggs, J. (1991). Approaches to learning in secondary and tertiary students in Hong Kong: Some comparative studies. Educational Research Journal, 6, 27-39.

Biggs, J., y Tang, C. (2011). Teaching for quality learning at university (4th Ed.). New York: McGraw Hill.

Biggs, J., Kember, D., y Leung, D. (2001). The Revised Two Factor Study Process Questionnaire: R-SPQ-2F. British Journal of Educational Psychology, 71, 133-149. doi: 10.1348/000709901158433

Byrne, M., Finlayson, O., Flood, B., Lyons, O. y Willis, P. (2010). A comparison of the learning approaches of accounting and science students at an Irish university. Journal of Further and Higher Education, 34(3), 369383. doi: 10.1080/0309877X.2010.484055 
Centro de Estudios de la Educación Argentina. (2015). Nuestra graduación universitaria es menor que la de nuestros vecinos Brasil y Chile. Recuperado de http:// www.ub.edu.ar/centros_de_estudio/cea/cea_numero_34.pdf

Cetin, B. (2015). Academic motivation and approaches to learning in predicting college students' academic achievement: Findings from Turkish and us samples. Journal of College and Teaching Learning, 12(2), 141150.

Cohen, J. (1977). Statistical power analysis for the behavioral science. New York: Academic Press.

Corengia, A., Pita, M., Mesurado, B. y Centeno, A. (2013). Predicting academic performance and attrition in undergraduate students. Liberabit, 19(1), 101-112.

Cornejo, C. y Saravia, J. (2014). Enfoques de aprendizaje, autodeterminación y estrategias metacognitivas en estudiantes de pedagogías de una universidad chilena. Ciencias Psicológicas, 8(1), 79-88.

Curry, L. (abril, 1983). An organization of learning styles theory and constructs. Paper presented at the 67th Anual Meeting of the American Educational Research Association. Montreal, Quebec.

De la Fuente, J., Sander, P. y Putwain, D. (2013). Relationship between undergraduate student confidence, approach to learning and academic performance: The role of gender. Revista de Psicodidáctica, 18(2), 375-393. doi: 10.1387/RevPsicodidact.7078

De Miguel, M. y Arias, J. (1999). La evaluación del rendimiento inmediato en la enseñanza universitaria. Revista de Educación, 320, 353-377.

Entwistle, N. (1988). Motivational factors in students' approaches to learning. En R. Schmeck (Ed.), Learning strategies and learning styles (pp. 21-51). New York: Plenum Press.

Entwistle, N. (1991). Approaches to learning and perceptions of the learning environment. Higher Education, 22, 201-204. doi: 10.1007/BF00132287

Entwistle, N. y Ramsden, P. (1983). Understanding student learning. London: Crrom Helm.

Finch, H. y French, B. (2013). A Monte Carlo comparison of robust MANOVA test statistics. Journal of Modern Applied Statistical Methods, 12(2), 35-81.

Freiberg-Hoffmann, A. y Fernández-Liporace, M. (2016). Enfoques de aprendizaje en estudiantes universitarios argentinos según el R-SPQ-2F: Análisis de sus propiedades psicométricas. Revista Colombiana de Psicología, 25(2), 307-329. doi: 10.15446/rcp.v25n2.51874
García-Berbén, A. (2005). Estudio de los enfoques de aprendizaje en estudiantes de Magisterio y Psicopedagogía. Electronic Journal of Research in Educational Psychology, 3(6), 109-126.

García de Fanelli, A. (2004). Indicadores y estrategias en relación con el abandono y la graduación universitarios. En Carlos Marquis (Ed.), La agenda universitaria (pp. 65-89). Buenos Aires: Colección Educación Superior, Universidad de Palermo.

Gargallo-López, B., Garfella-Esteban, P. y Pérez-Pérez, C. (2006). Enfoques de aprendizaje y rendimiento académico en estudiantes universitarios. Bordón, 58(3), 45-61.

Gobierno de Argentina-Ministerio de Educación. (10 de agosto de 1995). Ley de Educación Superior, Ley 24.52. Boletín Oficial $N^{\circ}$ 28.204. Buenos Aires.

Gobierno de Argentina-Ministerio de Educación-Secretaría de Políticas Universitarias. (2012). Anuario de Estadísticas Universitarias 2012. Buenos Aires: Ministerio de Educación. Recuperado de http://informacionpresupuestaria.siu.edu.ar/DocumentosSPU/diu/anuario_2012.pdf

Goikoetxea-Piérola, J., Martínez de Lahidalga, I. R. y Buján-Vidales, K. (2014). Enfoques de aprendizaje del alumnado universitario en función de los contextos de aula y curso. Contextos Educativos, 17, 9-21. doi: $10.18172 /$ con. 2590

González, F. (1997). Evidence of rote learning of science by Spanish university students. School Science and Mathematics, 97(8), 419-428. doi: 10.1111/j.1949-8594.1997. tb17387.x

Hasnor, H. Ahmad, Z. y Nordin, N. (2013). The relationship between learning approaches and academic achievement among Intec Students, Uitm Shah Alam. Procedia-Social and Behavioral Sciences, 90, 178-186. doi: 10.1016/j.sbspro.2013.07.080

Hemphill, J. (2003). Interpreting the magnitudes of correlation coefficients. American Psychologist, 58(1), 78-80. doi: 10.1037/0003-066X.58.1.78

Hernández-Pina, F., García-Sanz, M. y Maquilón-Sánchez, J. (2005). Análisis del Cuestionario de Procesos de Estudio-2 Factores de Biggs en estudiantes universitarios españoles. Revista Fuentes, 6, 96-114.

Huberty, C. y Petroskey, M. (2000). Multivariate analysis of variance and covariance. En Howard Tinsley and Steven Brown (Eds.), Handbook of applied multivariate statistics and mathematical modeling (pp. 183-208). New York: Academic Press. doi: 10.1016/ B978-012691360-6/50008-2 
IBM Corporation. (2012). IBM sPss Statistics (21). Recuperado de http://www-01.ibm.com/software/es/analytics/ spss/

Karagiannopoulou, E. y Milienos, F. (2015). Testing two path models to explore relationships between students' experiences of the teaching learning environment, approaches to learning and academic achievement. Educational Psychology, 35(1), 26-52. doi: 10.1080/01443410.2014.895800

Kember, D., Leung, D. y McNaught, C. (2008). A workshop activity to demonstrate that approaches to learning are influenced by the teaching and learning environment. Active Learning in Higher Education, 9(1), 43-56. doi: 10.1177/1469787407086745

Marrs, H. y Sigler, E. (2012). Male academic performance in college: The possible role of study strategies. Psychology of Men and Masculinity, 13(2), 227-241. doi: $10.1037 / \mathrm{a} 0022247$

Martín, E., García, L., Torbay, A. y Rodríguez, T. (2008). Estrategias de aprendizaje y rendimiento académico en estudiantes universitarios. International Journal of Psychology and Psychological Therapy, 8(3), 401-412.

Marton, F. (1976). What does it take to learn? Some implications of an alternative view of learning. En N. Entwistle (Ed.), Strategies for research and development in higher education (pp. 32-42). Amsterdam: Swets and Zeitlinger.

Marton, F. y Säljö, R. (1976). On qualitative differences in leraning-I: Outcome and process. British Journal of Educational Psychology, 46, 4-11. doi: 10.1111/j.20448279.1976.tb02980.x

Miles, J. y Banyard, P. (2007). Understanding and using statistics in psychology. California: SAGE Publications, Inc.

Mogre, V. y Amalba, A. (2014). Assessing the reliability and validity of the Revised Two Factor Study Process Questionnaire (R-SPQ-2F) in Ghanaian medical students. Journal of Educational Evaluation for Health Proffessions, 11. doi: 10.3352/jeehp.2014.11.19

Mogre, V. y Amalba, A. (2015). Approaches to learning among Ghanian students following a PBL-based medical curriculum. Education in Medicine Journal, 7(1), 38-44. doi: 10.5959/eimj.v7i1.319

Montero, Y., Pedroza, M., Astiz, M. y Vilanova, S. (2015). Caracterización de las actitudes de estudiantes universitarios de Matemática hacia los métodos numéricos. Revista Electrónica de Investigación Educativa, 17(1), 88-99.
Munshi, F., Al-Rukban, M. y Al-Hoqail, I. (2012). Reliability and validity of an arabic version of the Revised Two Factor Study Process Questionnaire R-SPQ-2F. Journal of Family and Community Medicine, 19(1), 33-37. doi: 10.4103/2230-8229.94010

Ng, G. y Ng, Y. (1997). Undergraduate students in a Computer Engineering course: A perspective of their learning approaches and motivation factor. Innovations in Education \& Training International, 34(1), 65-69. doi: $10.1080 / 1355800970340110$

Novak, J. (1977). A theory education. New York: Cornell University Press.

Núñez-Pérez, J. y González-Pienda, J. (1993). Determinantes del rendimiento académico. Oviedo: Universidad de Oviedo.

Organización de las Naciones Unidas para la Educación, la Ciencia y la Cultura. (2014). Enseñanza y aprendizaje. Lograr la calidad para todos. París: Unesco.

Parrino, M. (2008). ¿Evasión o expulsión? Los mecanismos de deserción en el primer año universitario (Tesis de doctorado). Universidad Nacional de Lanús, Argentina.

Pintos, M. E. (26 de marzo del 2012). En el primer año, el $58 \%$ de los estudiantes dejan o cambian de carrera. Clarín.com. Recuperado de http://www.clarin.com/ sociedad/primer-estudiantes-dejan-cambian-carrera_0_670732977.html

Rajaratnam, N., D’Cruz, S. y Chandrasekhar, M. (2013). Correlation between the learning approaches of first year medical students and their performance in multiple choice questions in Physiology. National Journal of Integrated Research in Medicine, 4(5), 43-48.

Romero-Medina, A., Hidalgo-Montesinos, M., González-Javier, F., Carrillo-Verdejo, E., Pedraja, M., García Sevilla, J. y Pérez-Sánchez, M. (2013). Enfoques de aprendizaje en estudiantes universitarios: comparación de resultados con los cuestionarios AssisT y R-SPQ-2F. Revista de Investigación Educativa, 31(2), 375-391. doi: 10.6018/ rie.31.2.151851

Sadlo, G. y Richardson, J. (2003). Approaches to studying and perceptions of the academic environment in students following problem-based and subject-based curricula. Higher Education Research and Development, 22, 253-274. doi: 10.1080/0729436032000145130

Salamonson, Y., Weaver, R., Chang, S., Koch, J., Bhathal, R., Khoo, C. y Wilson, I. (2013). Learning approaches as predictors of academic performance in first year health and science students. Nurse Education Today, 33(7), 729-733. doi: 10.1016/j.nedt.2013.01.013 
Salim, R. y Lotti de Santos, M. (2011). Evaluación de enfoques, motivaciones y estrategias de aprendizaje en estudiantes del primer año universitario de Odontología (UNT). Cuadernos de Educación, 9, 245-260.

Scheaffer, R., Mendenhall, W. y Ott, L. (2007). Elementos de muestreo. Madrid: Thomson.

Schmeck, R. (1983). Learning styles of college students. En R. Schmeck (Eds.), Individual differences in cognition (pp. 233-279). New York: Academic Press.

Seri, M., Goh, C., Mohd, H. y Seamah, R. (2010). The Bahasa Melayu R-SPQ-2F: A preliminary evidence of its validity. Procedia-Social and Behavioral Sciences, 7, 151-155. doi: 10.1016/j.sbspro.2010.10.022

Sevsen, C., Senol, D., Mehmet, K. y Ramazan, Y. (2013). Medical students approaches to learning and study skills. Procedia-Social and Behavioral Sciences, 93, 732736. doi: 10.1016/j.sbspro.2013.09.271

Smith, S.y Miller, R. (2005).Learning approaches: examination type, discipline of study, and gender. Educational Psychology, 25(1), 43-53. doi: 10.1080/0144341042000294886

Steinmann, A., Bosch, B. y Aiassa, D. (2013). Motivación y expectativas de los estudiantes por aprender ciencias en la universidad: un estudio exploratorio. Revista Mexicana de Investigación Educativa, 18(57), 585-598.

Stes, A., de Maeyer, S. y Van Petegem, P. (2013). Examining the cross-cultural sensitivity of the Revised Two Factor Study Process Questionnaire (R-SPQ-2F) and validation of dutch version. Plos One, 8(1). doi:10.1371/ journal.pone.0054099

Svedin, M., Balter, O., Scheja, M. y Pettersson, K. (2013). A surface approach to learning rewards first-year Engineering students. Learning and Teaching in Computing and Engineering. doi: 10.1109/LaTiCE.2013.40
Tabachnick, B. y Fidell, L. (2013). Using multivariate statistics. Boston: Pearson.

Tarabashkina, L. y Lietz, P. (2011). The impact of values and learning approaches on students achievement: Gender and academic discipline influences. Issues in Educational Reseach, 21(2), 210-231.

Vázquez-Martínez, A. y Alducin-Ochoa, J. (2014). Educational platforms and learning approaches in university education. Asian Social Science, 10(7). doi:10.5539/ass. v10n7p1

Ventura, A., Moscoloni, N. y Gagliardi, R. (2012). Estudio comparativo sobre los estilos de aprendizaje en estudiantes universitarios argentinos de diferentes disciplinas. Psicología desde el Caribe, 29(2), 276-304.

Wang, X., Su, Y., Cheung, S., Wong, E. y Kwong, T. (2013). An exploration of Biggs' constructive alignment in course design and its impact on students learning approaches. Assessment \& Evaluation in Higher Education, 38(4), 477-491. 10.1080/02602938.2012.658018

Wong, E., Kwong, T. y Thadani, D. (2014). The effects of students' perceptions of their learning experience on their approaches to learning: The learning experience inventory in courses (LEI-C). Education Journal, 3(6), 369-376. doi: 10.11648/j.edu.20140306.18

Zain, Z., Malan, I., Noordin, F. y Abdullah, Z. (2013). Assessing student approaches to learning: A case of business students at the Faculty of Business Management, UITM. Procedia-Socail and Behavioral Sciences, 90, 904913. doi: 10.1016/j.sbspro.2013.07.167

Zhang, L. (2000). University students' learning approaches in three cultures: An investigation of Biggs's 3P model. Psychology, 134(1),37-55.doi: 10.1080/00223980009600847 